\title{
Electroweak phase transition in the reduced minimal 3-3-1 model
}

\author{
Vo Quoc Phong* and Vo Thanh $\operatorname{Van}^{\dagger}$ \\ Department of Theoretical Physics, Ho Chi Minh City University of Science, Vietnam \\ Hoang Ngoc Long \\ Institute of Physics, Vietnamese Academy of Science and Technology, \\ 10 Dao Tan, Ba Dinh, Hanoi, Vietnam
}

(Dated: September 18, 2018)

\begin{abstract}
The electroweak phase transition is considered in framework of the reduced minimal 3-3-1 model (RM331). Structure of phase transition in this model is divided into two periods. The first period is the phase transition $S U(3) \rightarrow S U(2)$ at $\mathrm{TeV}$ scale and the second one is $S U(2) \rightarrow U(1)$, which is the like-Standard Model electroweak phase transition. When mass of the neutral Higgs boson $\left(h_{1}\right)$ is taken to be equal to the LHC value: $m_{h_{1}}=125 \mathrm{GeV}$, then these phase transitions are the first order phase transitions, the mass of $Z_{2}$ is about $4.8 \mathrm{TeV}$; and we find the region of parameter space with the first order phase transition at $v_{\rho_{0}}=246 \mathrm{GeV}$ scale, leading to an effective potential, where mass of the charged Higgs boson is in range of $3.258 \mathrm{TeV}<m_{h_{++}}<19.549 \mathrm{TeV}$. Therefore, with this approach, new bosons are the triggers of the first order electroweak phase transition with significant implications for the viability of electroweak baryogenesis scenarios.

PACS numbers: 11.15.Ex, 12.60.Fr, 98.80.Cq
\end{abstract}

Keywords: Spontaneous breaking of gauge symmetries, Extensions of electroweak Higgs sector, Particle-theory models (Early Universe)

\footnotetext{
*vqphong@hcmus.edu.vn

† vtvan@hcmus.edu.vn

$\ddagger$ hnlong@iop.vast.ac.vn
} 


\section{INTRODUCTION}

Electroweak phase transition (EWPT) is a type of symmetry-breaking phase transition which plays an important role at early stage of expanding universe. Particularly, the EWPT is important to explaining baryon asymmetry of our Universe. As proposed by Sakharov [1], three necessary conditions that a baryon-generating interaction in a theoretical model must satisfy to produce an excess of baryons over antibaryons are: baryon number violation, $\mathrm{C}$ and $\mathrm{CP}$ violations, and deviation from thermal equilibrium [1].

If baryon number (B) is conserved and is equal to zero, it will equal to zero forever. In contrast, B will be vanished in the state of thermal equilibrium. Therefore we need the third condition about the deviation from thermal equilibrium. The second condition is appropriate for ensuring a different decay rate for particles and antiparticles [1].

The baryon number, $\mathrm{C}$ and $\mathrm{CP}$ violations can be showed throughout the sphaleron rate and the CKM-matrix in models [2]. The sphaleron rate tells us about the baryon number violation and the non-zero phases of CKM-matrix tell us about CP violation.

It is well-known that in order to ensure sufficient the third condition, deviations from thermal equilibrium should be large enough, and therefore, the EWPT should be the first order phase transition. The EWPT is the transition between symmetric phase to asymmetric one in order to generate masses for particles. Therefore, the EWPT is related to the mass of the Higgs boson [1].

In the basic model of particles, the first and second conditions can be satisfied, but conditions on thermal imbalance is difficult to satisfy. So the analysis of the third condition is the only approach at present in order to explain the baryon asymmetry.

Why is the first order phase transition? The effective potential is a function of temperature and vacuum expectation values (VEVs). For very large temperature, it has only one minimum at the zero, and the symmetry is restored. As temperature goes to $T_{0}$, the nonzero second minimum appears, this is the sign of symmetry breaking. When temperature at the critical temperature $\left(T_{c}<T_{0}\right)$, the values of the effective potential at two minimums are equal together, the symmetry breaking is turned on. And at the critical temperature, if the two minimums are separated by a potential barrier, the phase transition will occur with bubble nucleations. Inside the bubbles, the scalar field stores a non-zero expectation value. If the bubble nucleation rate exceeds the universe's expansion rate, the bubbles collide and 
eventually fill all space [1]. Such a transition is called the first order phase transition. It is very violent and one can expect large deviations from thermal equilibrium [1]. The other possible scenario takes place if the two minimums are never separated by a potential barrier. In this case, the phase transition is a smooth transition, not violent or the second order phase transition.

To study the EWPT, ones consider the hight-temperature effective potential as follows

$$
V_{e f f}=D \cdot\left(T^{2}-T_{0}^{\prime 2}\right) v^{2}-E \cdot T v^{3}+\frac{\lambda_{T}}{4} v^{4},
$$

where $v$ is the VEV of Higgs. In order to have the first order phase transition, the strength of phase transition should be larger than the unit, i.e., $\frac{v_{c}}{T_{c}} \geq 1$.

The EWPT has been investigated in the Standard Model (SM) [1, 2] and in various extended models 3 -10]. Also, a very interesting research has showed that dark matter as triggers of the electroweak phase transition [11]. For the SM, the strength of the EWPT is larger than the unit at the electroweak scale, appears too weak for the experimentally allowed mass of the SM scalar Higgs boson [1, 2]; therefore, it seems that electroweak baryogenesis requires a new physics beyond the SM at weak scale [3].

Before the neutral Higgs was found, the study of phase transitions in most of models has been focused on two basic issues: determining the order of phase transition and the neutral Higgs mass. For the SM, the first order phase transition problem has one variable which is the mass of the neutral Higgs boson. However, for the extended models, this problem has at least two variables, the first one is the Higgs mass and the rest includes the masses of heavy particles. Recently, the neutral Higgs has been discovered by the LHC [12], so the electroweak phase transition problem is reduced by one variable. This gives a lot of hope for the extended models in examining the EWPT. The remarkable successes of the last survey are:

- The SM cannot explain this phenomenon: sources of $\mathrm{CP}$ violation is smaller than Baryon Asymmetry of Universe (BAU) and no the first order phase transition with the large mass of the neutral Higgs or other word speaking the SM is not enough triggers for the first order phase transition to turn on [1].

- The extended models such as the two Higgs doublet model (TDHM) or MSSM, can explain the baryon asymmetry, because sources of $\mathrm{CP}$ violation in these models are 
stronger than in the SM and they have the first order phase transition with the mass of neutral Higgs about $120 \mathrm{GeV}$. Triggers for the first order phase transition in these models are heavy bosons, or dark matter candidates [7-9, 11].

Among the extended models, the models based on $\mathrm{SU}(3)_{C} \otimes \mathrm{SU}(3)_{L} \otimes \mathrm{U}(1)_{X}$ gauge group (called 3-3-1 for short) [13, 15] have some intriguing features such as they can answer on the generation problem [13, 15], provide the electric charge quantization [16], etc. According to the above summarizing, we hope that the 3-3-1 models can also answer the problem of baryon asymmetry in our Universe.

The current 3-3-1 models have many different forms, but the core is based on the above mentioned gauge group. The most disadvantage of the 3-3-1 models is the complication in the Higgs sector, namely these models need at least three Higgs triplets to generate masses of fermions. Recently there are attempts to solve this problem, and some models with the simplest Higgs sector (with only two $\mathrm{SU}(3)_{L}$ Higgs triplets) have been constructed.

With such a group structure, the 3-3-1 models must have at least two Higgs triplets [17, 18]. Therefore, the number of bosons in the 3-3-1 models will many more than in the SM and symmetry breaking structure is different to the SM.

In the present work, we consider the EWPT in the reduced minimal 3-3-1 (RM331) model [18] because of its simplicity. This model has the minimal leptonic content (with only the SM leptons) and the presence of bileptons: a singly and a doubly charged gauge bosons, $V^{ \pm}$and $U^{ \pm \pm}$, the heavy neutral boson $Z_{2}$ and the exotic quarks. This model also has two Higgs triplets. Therefore, the physical scalar spectrum of the RM331 model is composed by a doubly charged scalar $h^{++}$and two neutral scalars $h_{1}$ and $h_{2}$ [18]. These new particles and exotic quarks can be triggers for the first order phase transition.

The plan of the paper is as follows. In Sec II we give a review of the RM331 about the boson, lepton and Higgs sectors. In Sec. III, we find the effective potential in the RM331 that has the contribution from heavy bosons and a contribution part like in the SM. In Sec. IV, we calculate in details structure of phase transition in the RM331, find the first order phase transition and show constraints on mass of charged Higgs boson. Finally, we summarize and make outlooks in Sec. D. 


\section{A REVIEW OF THE RM331 MODEL}

The fermion content of the RM331 model is the same as of the minimal 3-3-1 model [13]. The difference is only in the Higgs sector.

\section{A. Higgs potential}

The Higgs potential in the RM331 [18] is given by

$$
\begin{aligned}
V(\chi, \rho)= & \mu_{1}^{2} \rho^{\dagger} \rho+\mu_{2}^{2} \chi^{\dagger} \chi+\lambda_{1}\left(\rho^{\dagger} \rho\right)^{2}+\lambda_{2}\left(\chi^{\dagger} \chi\right)^{2} \\
& +\lambda_{3}\left(\rho^{\dagger} \rho\right)\left(\chi^{\dagger} \chi\right)+\lambda_{4}\left(\rho^{\dagger} \chi\right)\left(\chi^{\dagger} \rho\right) .
\end{aligned}
$$

The scalar sector contains only two Higgs scalar triplets [18]

$$
\rho=\left(\begin{array}{c}
\rho^{+} \\
\rho^{0} \\
\rho^{++}
\end{array}\right) \sim(\mathbf{3}, 1), \quad \chi=\left(\begin{array}{c}
\chi^{-} \\
\chi^{--} \\
\chi^{0}
\end{array}\right) \sim(\mathbf{3},-1) .
$$

Expansion of $\rho^{0}$ and $\chi^{0}$ around their VEVs is usually

$$
\rho^{0}, \chi^{0} \rightarrow \frac{1}{\sqrt{2}}\left(v_{\rho, \chi}+R_{\rho, \chi}+i I_{\rho, \chi}\right) .
$$

This potential immediately gives us two charged Goldstone bosons $\rho^{ \pm}$and $\chi^{ \pm}$which are eaten by the gauge bosons $W^{ \pm}$and $V^{ \pm}$.

Let us resume content of the Higgs sector: the physical scalar spectrum of the RM331 model is composed by a doubly charged scalar $h^{++}$and two neutral scalars $h_{1}$ and $h_{2}$. Since the lightest neutral field, $h_{1}$, is basically a $S U(2)_{L}$ component, we identify it as the SM Higgs boson. In the effective limit $v_{\chi} \gg v_{\rho}$, the Higgs content can be summarized as follows

$$
\rho=\left(\begin{array}{c}
G_{W^{+}} \\
\frac{v_{\rho}}{\sqrt{2}}+\frac{1}{\sqrt{2}}\left(h_{1}+i G_{Z}\right) \\
h^{++}
\end{array}\right), \quad \chi=\left(\begin{array}{c}
G_{V^{-}} \\
G_{U^{--}} \\
\frac{v_{\chi}}{\sqrt{2}}+\frac{1}{\sqrt{2}}\left(h_{2}+i G_{Z^{\prime}}\right)
\end{array}\right),
$$

where the Higgs masses are given by:

$$
\begin{aligned}
M_{h_{1}}^{2} & =\left(\lambda_{1}-\frac{\lambda_{3}^{2}}{4 \lambda_{2}}\right) v_{\rho}^{2}, \quad M_{h_{2}}^{2}=\lambda_{2} v_{\chi}^{2}+\frac{\lambda_{3}^{2}}{4 \lambda_{2}} v_{\rho}^{2}, \\
M_{h^{--}}^{2} & =\frac{\lambda_{4}}{2}\left(v_{\chi}^{2}+v_{\rho}^{2}\right) .
\end{aligned}
$$




\section{B. Gauge boson sector}

The masses of gauge bosons appear in the Lagrangian part

$$
\mathcal{L}=\left(\mathcal{D}_{\mu} \chi\right)^{\dagger}\left(\mathcal{D}^{\mu} \chi\right)+\left(\mathcal{D}_{\mu} \rho\right)^{\dagger}\left(\mathcal{D}^{\mu} \rho\right)
$$

where

$$
\mathcal{D}_{\mu}=\partial_{\mu}-i g A_{\mu}^{a} \frac{\lambda^{a}}{2}-i g_{X} X \frac{\lambda_{9}}{2} B_{\mu},
$$

with $\lambda_{9}=\sqrt{\frac{2}{3}} \operatorname{diag}(1,1,1)$ so $\operatorname{Tr}\left(\lambda_{9} \lambda_{9}\right)=2$. The couplings of $S U(3)_{L}$ and $U(1)_{X}$ satisfy the relation:

$$
\frac{g_{X}^{2}}{g^{2}}=\frac{6 s_{W}^{2}}{1-4 s_{W}^{2}},
$$

where $c_{W}=\cos \theta_{W}, s_{W}=\sin \theta_{W}, t_{W}=\tan \theta_{W}$ with $\theta_{W}$ is the Weinberg angle.

Substitution of the expansion in Eq. (3) into (7) leads to the following result

$$
\begin{aligned}
& W^{ \pm}=\frac{A^{1} \mp i A^{2}}{\sqrt{2}} \rightarrow m_{W^{ \pm}}^{2}=\frac{g^{2} v_{\rho}^{2}}{4}, \\
& V^{ \pm}=\frac{A^{4} \pm i A^{5}}{\sqrt{2}} \rightarrow m_{V^{ \pm}}^{2}=\frac{g^{2} v_{\chi}^{2}}{4}, \\
& U^{ \pm \pm}=\frac{A^{6} \pm i A^{7}}{\sqrt{2}} \rightarrow m_{U^{ \pm \pm}}^{2}=\frac{g^{2}\left(v_{\rho}^{2}+v_{\chi}^{2}\right)}{4}
\end{aligned}
$$

From (9), it follows that $v_{\rho}=246 \mathrm{GeV}$ and the relation

$$
m_{U}^{2}-m_{V}^{2}=m_{W}^{2}
$$

In the neutral gauge boson sector, with the basis $\left(A_{\mu}^{3}, A_{\mu}^{8}, B_{\mu}\right)$, mass matrix is given by

$$
M^{2}=\frac{g^{2}}{4}\left(\begin{array}{ccc}
v_{\rho}^{2} & -\frac{v_{\rho}^{2}}{\sqrt{3}} & -2 \kappa v_{\rho}^{2} \\
-\frac{v_{\rho}^{2}}{\sqrt{3}} & \frac{1}{3}\left(v_{\rho}^{2}+4 v_{\chi}^{2}\right) & \frac{2}{\sqrt{3}}\left(v_{\rho}^{2}+2 v_{\chi}^{2}\right) \\
-2 \kappa v_{\rho}^{2} & \frac{2}{\sqrt{3}}\left(v_{\rho}^{2}+2 v_{\chi}^{2}\right) & 4 \kappa^{2}\left(v_{\rho}^{2}+v_{\chi}^{2}\right),
\end{array}\right),
$$

where $\kappa=\frac{g_{X}}{g}$. We can easily identify the photon field $A_{\mu}$ as well as the massive neutral $Z$ and $Z^{\prime}$ bosons [19]

$$
\begin{aligned}
& A_{\mu}=s_{W} A_{\mu}^{3}+c_{W}\left(\sqrt{3} t_{W} A_{\mu}^{8}+\sqrt{1-3 t_{W}^{2}} B_{\mu}\right), \\
& Z_{\mu}=c_{W} A_{\mu}^{3}-s_{W}\left(\sqrt{3} t_{W} A_{\mu}^{8}+\sqrt{1-3 t_{W}^{2}} B_{\mu}\right),
\end{aligned}
$$

and

$$
Z_{\mu}^{\prime}=-\sqrt{1-3 t_{W}^{2}} A_{\mu}^{8}+\sqrt{3} t_{W} B_{\mu}
$$


where the mass-square matrix for $\left\{Z, Z^{\prime}\right\}$ is given by

$$
\left(\begin{array}{cc}
m_{Z}^{2} & m_{Z Z^{\prime}}^{2} \\
m_{Z Z^{\prime}}^{2} & m_{Z^{\prime}}^{2}
\end{array}\right)
$$

with

$$
\begin{aligned}
m_{Z}^{2} & =\frac{1}{4} \frac{g^{2}}{\cos ^{2} \theta_{W}} v_{\rho}^{2}, \\
m_{Z^{\prime}}^{2} & =\frac{1}{3} g^{2}\left[\frac{\cos ^{2} \theta_{W}}{1-4 \sin ^{2} \theta_{W}} v_{\chi}^{2}+\frac{1-4 \sin ^{2} \theta_{W}}{4 \cos ^{2} \theta_{W}} v_{\rho}^{2}\right], \\
m_{Z Z^{\prime}}^{2} & =\frac{1}{4 \sqrt{3}} g^{2} \frac{\sqrt{1-4 \sin ^{2} \theta_{W}}}{\cos ^{2} \theta_{W}} v_{\rho}^{2} .
\end{aligned}
$$

Diagonalizing the mass matrix gives the mass eigenstates $Z_{1}$ and $Z_{2}$ which can be taken as mixtures

$$
\begin{aligned}
& Z_{1}=Z \cos \phi-Z^{\prime} \sin \phi, \\
& Z_{2}=Z \sin \phi+Z^{\prime} \cos \phi .
\end{aligned}
$$

The mixing angle $\phi$ is given by

$$
\tan 2 \phi=\frac{m_{Z}^{2}-m_{Z_{1}}^{2}}{m_{Z_{2}}^{2}-m_{Z}^{2}}
$$

where $m_{Z_{1}}$ and $m_{Z_{2}}$ are the physical mass eigenvalues

$$
\begin{aligned}
& m_{Z_{1}}^{2}=\frac{1}{2}\left\{m_{Z^{\prime}}^{2}+m_{Z}^{2}-\left[\left(m_{Z^{\prime}}^{2}-m_{Z}^{2}\right)^{2}-4\left(m_{Z Z^{\prime}}^{2}\right)^{2}\right]^{1 / 2}\right\}, \\
& m_{Z_{2}}^{2}=\frac{1}{2}\left\{m_{Z^{\prime}}^{2}+m_{Z}^{2}+\left[\left(m_{Z^{\prime}}^{2}-m_{Z}^{2}\right)^{2}-4\left(m_{Z Z^{\prime}}^{2}\right)^{2}\right]^{1 / 2}\right\} .
\end{aligned}
$$

In diagonalization, the mass of $Z_{1}$ is approximately proportional to $v_{\rho}$ (because $v_{\chi} \gg v_{\rho}$ ), so $Z_{1}$ likes the neutral gauge boson $Z$ in the SM. The mass of the new heavy boson $Z_{2}$ depends on $v_{\rho}$ and $v_{\chi}$; in addition, $m_{W}=80.39 \mathrm{GeV}$ and $v_{\rho_{0}}=246 \mathrm{GeV}[20]$. Choosing $v_{\chi 0}=4 \mathrm{TeV}$ [14, 18], we obtain $m_{V}=1307.15 \mathrm{GeV}$ and $m_{U}=1309.62 \mathrm{GeV}$. If we choose $s_{W}^{2}=0.23116$ [20], we derive $m_{Z_{1}} \approx m_{Z}=91.68 \mathrm{GeV}$ and $m_{Z_{2}}=4.821 \mathrm{TeV}$. Hence we can approximate $m_{Z_{2}} \approx 1.2 v_{\chi}$.

\section{Fermion sector}

The fermion sector in the model under consideration is the same as in the minimal 3-3-1 model [13]. The Yukawa couplings give the exotic quark masses [18]

$$
L_{Y u k}^{e x o t}=\lambda_{11}^{T} \bar{Q}_{1 L} \chi T_{R}+\lambda_{i j}^{D} \bar{Q}_{i L} \chi^{*} D_{j R}+\text { H.c. }
$$




$$
\begin{aligned}
= & \lambda_{11}^{T}\left(\bar{u}_{1 L} \chi^{-}+\bar{d}_{1 L} \chi^{--}+\bar{T}_{L} \chi^{0}\right) T_{R} \\
& +\lambda_{i j}^{D}\left(\bar{d}_{i L} \chi^{+}-\bar{u}_{i L} \chi^{++}+\bar{D}_{i L} \chi^{0 *}\right) D_{j R}+\text { H.c. }
\end{aligned}
$$

When the $\chi$ field develops its VEV, these couplings lead to the mass matrix in the basis $\left(T, D_{2}, D_{3}\right)$

$$
M_{J}=\frac{v_{\chi}}{\sqrt{2}}\left(\begin{array}{ccc}
\lambda_{11}^{T} & 0 & 0 \\
0 & \lambda_{22}^{D} & \lambda_{23}^{D} \\
0 & \lambda_{32}^{D} & \lambda_{33}^{D}
\end{array}\right)
$$

So the exotic quarks have masses around few $\mathrm{TeVs}$ because their masses are proportional to $v_{\chi}$. Therefore we see that masses of exotic quarks approximately equal to $m_{Z_{2}}$, but they only involve in the transition phase $S U(3) \rightarrow S U(2)$.

For usual quarks, the Yukawa couplings give the masses of them through the triplet $\rho$. Therefore, also like the SM, the usual quarks only involve in the transition phase $S U(2) \rightarrow$ $U(1)$.

However, the charged lepton masses arise from the effective dimension five operator through the couplings of both $\chi$ and $\rho$ with the following Lagrangian [18]

$$
L_{Y u k}^{l}=\frac{\kappa_{l}}{\Lambda}\left(\overline{f_{L}^{c}} \rho^{*}\right)\left(\chi^{\dagger} f_{L}\right)+H . c .
$$

From Lagrangian (11), we obtain $m_{l}=\frac{v_{\chi}}{\Lambda} \kappa_{l} v_{\rho}$, the coupling constant $v_{\chi} / \Lambda \approx 1$, so $m_{l} \approx \kappa_{l} v_{\rho}$. Finally the masses of charged leptons depend only on $v_{\rho}$. Therefore, they only involve in the transition phase $S U(2) \rightarrow U(1)$. Taking into account $m_{e}=0.5 \mathrm{MeV}$, $m_{\mu}=105 \mathrm{MeV}, m_{\tau}=1.77 \mathrm{GeV}$, ones get $k_{e}=2 \times 10^{-5}, k_{\mu}=4.3 \times 10^{-3}$ and $k_{\tau}=7.2 \times 10^{-2}$.

\section{EFFECTIVE POTENTIAL IN RM331}

From the Higgs potential we obtain $V_{0}$ depending on VEVs as follows

$$
V_{0}\left(v_{\chi}, v_{\rho}\right)=\mu_{1}^{2} v_{\chi}^{2}+\mu_{2}^{2} v_{\rho}^{2}+\lambda_{1} v_{\chi}^{4}+\lambda_{2} v_{\rho}^{4}+\lambda_{3} v_{\chi}^{2} v_{\rho}^{2}
$$

Here $V_{0}$ has quartic form like in the SM, but it depends on two variables $v_{\chi}$ and $v_{\rho}$ and has the mixing between $v_{\chi}$ and $v_{\rho}$. However, developing the potential (1), we obtain two minimum equations. Therefore, we can transform the mixing between $v_{\chi}$ and $v_{\rho}$ to the form that depends only on $v_{\chi}$ or $v_{\rho}$. Hence, we can write $V_{0}\left(v_{\chi}, v_{\rho}\right)=V_{0}\left(v_{\chi}\right)+V_{0}\left(v_{\rho}\right)$. 
In order to derive effective potential, starting from the Higgs Lagrangian and using the principle of least action, we arrive at the equation of motion for fields. Expanding Higgs fields around VEVs and averaging over space for all fields, we obtain the one-loop effective potential (for details, see Ref.[1]).

The full Higgs Lagrangian in the RM331 model is given by

$$
\mathcal{L}=\left(\mathcal{D}_{\mu} \chi\right)^{\dagger}\left(\mathcal{D}^{\mu} \chi\right)+\left(\mathcal{D}_{\mu} \rho\right)^{\dagger}\left(\mathcal{D}^{\mu} \rho\right)+V(\chi, \rho),
$$

where

$$
\begin{aligned}
V(\chi, \rho)= & \mu_{1}^{2} \rho^{\dagger} \rho+\mu_{2}^{2} \chi^{\dagger} \chi+\lambda_{1}\left(\rho^{\dagger} \rho\right)^{2}+\lambda_{2}\left(\chi^{\dagger} \chi\right)^{2} \\
& +\lambda_{3}\left(\rho^{\dagger} \rho\right)\left(\chi^{\dagger} \chi\right)+\lambda_{4}\left(\rho^{\dagger} \chi\right)\left(\chi^{\dagger} \rho\right) .
\end{aligned}
$$

Expanding $\rho$ and $\chi$ around $v_{\rho}$ and $v_{\chi}$ which are considered as variables [24], so we obtain

$$
\mathcal{L}=\frac{1}{2} \partial^{\mu} v_{\chi} \partial_{\mu} v_{\chi}+\frac{1}{2} \partial^{\mu} v_{\rho} \partial_{\mu} v_{\rho}+V_{0}\left(v_{\chi}, v_{\rho}\right)+\sum_{\text {boson }} m_{\text {boson }}^{2}\left(v_{\chi}, v_{\rho}\right) W^{\mu} W_{\mu},
$$

where $W$ runs over all gauge fields and Higgs bosons. Through the boson mass formulations as in the above sections, we can split masses of particles into two parts as follows

$$
m_{\text {boson }}^{2}\left(v_{\chi}, v_{\rho}\right)=m_{\text {boson }}^{2}\left(v_{\chi}\right)+m_{\text {boson }}^{2}\left(v_{\rho}\right) .
$$

The effective potential is the function that depends on VEVs and temperature. The masses of particles depend on VEV of Higgs bosons. Therefore, when we consider the effective potential, we must consider contributions from fermions and bosons. However, for fermions, we have retained here only the top quark and exotic quarks, which dominate over the contributions from the other fermions [1]. And in the RM331 model, there are two VEVS so we have two motion equations according to $v_{\chi}$ and $v_{\rho}$

$$
\begin{aligned}
& \partial^{\mu} v_{\chi} \partial_{\mu} v_{\chi}+\frac{\partial V_{0}\left(v_{\chi}\right)}{\partial v_{\chi}}+\sum \frac{\partial m_{\text {bosons }}^{2}\left(v_{\chi}\right)}{\partial v_{\chi}} W^{\mu} W_{\mu}+\sum \frac{\partial m_{\text {exotic-quarks }}\left(v_{\chi}\right)}{\partial v_{\chi}} Q \bar{Q}=0 \\
& \partial^{\mu} v_{\rho} \partial_{\mu} v_{\rho}+\frac{\partial V_{0}\left(v_{\rho}\right)}{\partial v_{\rho}}+\sum \frac{\partial m_{\text {bosons }}^{2}\left(v_{\rho}\right)}{\partial v_{\rho}} W^{\mu} W_{\mu}+\frac{\partial m_{\text {top-quark }}\left(v_{\rho}\right)}{\partial v_{\rho}} t \bar{t}=0 .
\end{aligned}
$$

The RM331 has the gauge bosons: two massive like the SM bosons $Z_{1}$ and $W^{ \pm}$and the new heavy neutral boson $Z_{2}$, the singly and doubly charged gauge bosons $U^{ \pm \pm}$and $V^{ \pm}$; and two doubly charged Higgses $h^{++}$and $h^{--}$, one heavy neutral Higgs $h_{2}$ and one like-SM Higgs $h_{1}$. Masses of gauge bosons and Higgses in the RM 331 model are presented in Table [1] 
TABLE I: Mass formulations of bosons in the RM331 model

\begin{tabular}{|l|l|c|c|}
\hline Bosons & $m^{2}\left(v_{\chi}, v_{\rho}\right)$ & $m^{2}\left(v_{\chi}\right)$ & $m^{2}\left(v_{\rho}\right)$ \\
\hline$m_{W^{ \pm}}^{2}$ & $\frac{g^{2} v_{\rho}^{2}}{4}$ & 0 & $80.39^{2}(\mathrm{GeV})^{2}$ \\
\hline$m_{V^{ \pm}}^{2}$ & $\frac{g^{2} v_{\chi}^{2}}{4}$ & $1307.15^{2}(\mathrm{GeV})^{2}$ & 0 \\
\hline$m_{U^{ \pm \pm}}^{2}$ & $\frac{g^{2}\left(v_{\rho}^{2}+v_{\chi}^{2}\right)}{4}$ & $1307.15^{2}(\mathrm{GeV})^{2}$ & $80.39^{2}(\mathrm{GeV})^{2}$ \\
\hline$m_{Z_{1}}^{2} \sim m_{Z}^{2}$ & $\frac{1}{4} \frac{g^{2}}{\cos ^{2} \theta_{W}} v_{\rho}^{2}$ & 0 & $91.682^{2}(\mathrm{GeV})^{2}$ \\
\hline$m_{Z_{2}}^{2} \sim m_{Z^{\prime}}^{2}$ & $\frac{1}{3} g^{2}\left[\frac{\cos ^{2} \theta_{W}}{1-4 \sin ^{2} \theta_{W}} v_{\chi}^{2}+\frac{1-4 \sin ^{2} \theta_{W}}{4 \cos ^{2} \theta_{W}} v_{\rho}^{2}\right.$ & $4.8^{2}(\mathrm{TeV})^{2}$ & $14.53^{2}(\mathrm{GeV})^{2}$ \\
\hline$m_{h_{1}}^{2}$ & $\left(\lambda_{1}-\frac{\lambda_{3}^{2}}{4 \lambda_{2}}\right) v_{\rho}^{2}$ & 0 & $125^{2}(\mathrm{GeV})^{2}$ \\
\hline$m_{h^{--}}^{2}$ & $\frac{\lambda_{4}}{2}\left(v_{\chi}^{2}+v_{\rho}^{2}\right)$ & $\frac{\lambda_{4}}{2} v_{\chi}^{2}$ & $\frac{\lambda_{4}}{2} v_{\rho}^{2}$ \\
\hline$m_{h_{2}}^{2}$ & $\lambda_{2} v_{\chi}^{2}+\frac{\lambda_{3}^{2}}{4 \lambda_{2}} v_{\rho}^{2}$ & $\lambda_{2} v_{\chi}^{2}$ & $\frac{\lambda_{3}^{2}}{4 \lambda_{2}} v_{\rho}^{2}$ \\
\hline
\end{tabular}

From two equations (12) and (13) and averaging over space by using Bose-Einstein and Fermi-Dirac distributions for bosons and fermions, we obtain the following effective potentials

$$
\begin{aligned}
V_{e f f}\left(v_{\chi}\right)= & V_{0}\left(v_{\chi}\right)+\frac{3}{64 \pi^{2}}\left(m_{Z_{2}}^{4}\left(v_{\chi}\right) \ln \frac{m_{Z_{2}}^{2}\left(v_{\chi}\right)}{Q^{2}}-12 m_{Q}^{4}\left(v_{\chi}\right) \ln \frac{m_{Q}^{2}\left(v_{\chi}\right)}{Q^{2}}\right) \\
& +\frac{1}{64 \pi^{2}}\left(m_{h_{2}}^{4}\left(v_{\chi}\right) \ln \frac{m_{h_{2}}^{2}\left(v_{\chi}\right)}{Q^{2}}+2 m_{h^{++}}^{4}\left(v_{\chi}\right) \ln \frac{m_{h^{++}}^{2}\left(v_{\chi}\right)}{Q^{2}}\right) \\
& +\frac{3}{64 \pi^{2}}\left(2 m_{U}^{4}\left(v_{\chi}\right) \ln \frac{m_{U}^{2}\left(v_{\chi}\right)}{Q^{2}}+2 m_{V}^{4}\left(v_{\chi}\right) \ln \frac{m_{V}^{2}\left(v_{\chi}\right)}{Q^{2}}\right) \\
& +\frac{T^{4}}{4 \pi^{2}}\left[F_{-}\left(\frac{m_{h_{2}}\left(v_{\chi}\right)}{T}\right)+2 F_{-}\left(\frac{m_{h^{++}}\left(v_{\chi}\right)}{T}\right)+12 F_{+}\left(\frac{m_{Q}\left(v_{\chi}\right)}{T}\right)\right] \\
& +\frac{3 T^{4}}{4 \pi^{2}}\left[F_{-}\left(\frac{m_{Z_{2}}\left(v_{\chi}\right)}{T}\right)+2 F_{-}\left(\frac{m_{U}\left(v_{\chi}\right)}{T}\right)+2 F_{-}\left(\frac{m_{V}\left(v_{\chi}\right)}{T}\right)\right]
\end{aligned}
$$

and

$$
\begin{aligned}
V_{e f f}\left(v_{\rho}\right)= & V_{0}\left(v_{\rho}\right)+\frac{3}{64 \pi^{2}}\left(m_{Z_{1}}^{4}\left(v_{\rho}\right) \ln \frac{m_{Z_{1}}^{2}\left(v_{\rho}\right)}{Q^{2}}+m_{Z_{2}}^{4}\left(v_{\rho}\right) \ln \frac{m_{Z_{2}}^{2}\left(v_{\rho}\right)}{Q^{2}}\right. \\
& \left.+2 m_{W}^{4}\left(v_{\rho}\right) \ln \frac{m_{W}^{2}\left(v_{\rho}\right)}{Q^{2}}+2 m_{U}^{4}\left(v_{\rho}\right) \ln \frac{m_{U}^{2}\left(v_{\rho}\right)}{Q^{2}}-4 m_{t}^{4}\left(v_{\rho}\right) \ln \frac{m_{t}^{2}\left(v_{\rho}\right)}{Q^{2}}\right) \\
& +\frac{1}{64 \pi^{2}}\left(m_{h_{1}}^{4}\left(v_{\rho}\right) \ln \frac{m_{h_{1}}^{2}\left(v_{\rho}\right)}{Q^{2}}-m_{h_{2}}^{4}\left(v_{\rho}\right) \ln \frac{m_{h_{2}}^{2}\left(v_{\rho}\right)}{Q^{2}}+2 m_{h^{++}}^{4} \ln \frac{m_{h^{++}}^{2}\left(v_{\rho}\right)}{Q^{2}}\right) \\
& +\frac{T^{4}}{4 \pi^{2}}\left[F_{-}\left(\frac{m_{h_{1}}\left(v_{\rho}\right)}{T}\right)-F_{-}\left(\frac{m_{h_{2}}\left(v_{\rho}\right)}{T}\right)+2 F_{-}\left(\frac{m_{h^{++}}\left(v_{\rho}\right)}{T}\right)\right]
\end{aligned}
$$




$$
\begin{aligned}
& +\frac{3 T^{4}}{4 \pi^{2}}\left[4 F_{+}\left(\frac{m_{t}\left(v_{\rho}\right)}{T}\right)+F_{-}\left(\frac{m_{Z_{1}}\left(v_{\rho}\right)}{T}\right)+F_{-}\left(\frac{m_{Z_{2}}\left(v_{\rho}\right)}{T}\right)\right. \\
& \left.+2 F_{-}\left(\frac{m_{W}\left(v_{\rho}\right)}{T}\right)+2 F_{-}\left(\frac{m_{U}\left(v_{\rho}\right)}{T}\right)\right],
\end{aligned}
$$

where

$$
\begin{aligned}
F_{\mp}\left(\frac{m_{\phi}}{T}\right) & =\int_{0}^{\frac{m_{\phi}}{T}} \alpha J_{\mp}^{(1)}(\alpha, 0) d \alpha, \\
J_{\mp}^{(1)}(\alpha, 0) & =2 \int_{\alpha}^{\infty} \frac{\left(x^{2}-\alpha^{2}\right)^{1 / 2}}{e^{x} \mp 1} d x .
\end{aligned}
$$

The total effective potential in the RM331 model can be rewritten as follows

$$
V_{e f f}^{R M 331}=V_{e f f}\left(v_{\chi}\right)+V_{e f f}\left(v_{\rho}\right)
$$

\section{ELECTROWEAK PHASE TRANSITION}

The symmetry breaking in the RM331 can take place sequentially: due to two scales of symmetry breaking which are very different, $v_{\chi_{0}} \gg v_{\rho_{0}}\left(v_{\chi_{0}} \sim 4-5 \mathrm{TeV}\right.$ [14, 18], $v_{\rho_{0}}=246$ $\mathrm{GeV})$ and because of the accelerating universe. The symmetry breaking $S U(3) \rightarrow S U(2)$ takes place before the symmetry breaking $S U(2) \rightarrow U(1)$.

The symmetry breaking $S U(3) \rightarrow S U(2)$ through $\chi_{0}$, generates masses of the heavy gauge bosons such as $U^{ \pm \pm}, V^{ \pm}, Z_{2}$, and the exotic quarks. Therefore, the phase transition $S U(3) \rightarrow S U(2)$ only depends on $v_{\chi}$. When our universe has been expanding and cooling due to $v_{\rho_{0}}$ scale, the symmetry breaking or phase transition $S U(2) \rightarrow U(1)$ is turned on through $\rho_{0}$, which generates masses of the SM particles and the last part of masses of $U^{ \pm \pm}$. Therefore phase transition $S U(2) \rightarrow U(1)$ only depends on $v_{\rho}$. For the current universe having the baryon asymmetry, this asymmetry must exist from the initial conditions of the universe. If the early universe has not the baryon asymmetry, the current universe will not have it [1]. In other words, this asymmetry exists throughout periods of the universe to date. In order to describe baryogenesis, models must satisfy three conditions given by Sakharov. Especially in the third condition, the spontaneous symmetry breaking must be associated with the first order phase transition. In the RM331 model, the spontaneous symmetry breaking takes place in two different energy scales or other word speaking the electroweak phase transition is the combination of two different phase transitions. Therefore, if the RM331 model well describes this phenomenon, both two phase transitions are the first order phase transition. 
In contrast, if one of two phase transitions is not the first order phase transition, the RM331 model will not fully describe this asymmetry, since this model does not ensure the continuity of baryogenesis in the universe.

Through the boson mass formulations in the above sections, we see that boson $V^{ \pm}$only involves in the first phase transition $S U(3) \rightarrow S U(2)$. The gauge bosons $Z_{1}, W^{ \pm}$and $h_{1}$ only involve in the second phase transition $S U(2) \rightarrow U(1)$. However, $U^{ \pm \pm}, Z_{2}$ and $h^{--}$ involve in both two phase transitions.

With that structure of phase transition, we see that the mass of $U^{ \pm \pm}$, is generated by both two phase transitions. When the universe at $v_{\chi_{0}}$ scale, the symmetry breaking $S U(3) \rightarrow S U(2)$ generates masses for the exotic quarks and a part of $U^{ \pm \pm}$, or other word speaking, it eaten one Goldstone boson $\chi^{ \pm \pm}$of triplet $\chi$. When the universe cool to $v_{\rho_{0}}$ scale, the symmetry breaking $S U(2) \rightarrow U(1)$ is turned on, it generates masses for the SM particles and the last part of $U^{ \pm \pm}$, or $U^{ \pm \pm}$eaten the other Goldstone boson $\rho^{ \pm \pm}$of triplet $\rho$.

\section{A. Phase transition $S U(3) \rightarrow S U(2)$}

This phase transition involves exotic quarks, heavy bosons, without involvement of the SM particles, the effective potential of the EWPT $S U(3) \rightarrow S U(2)$ is $V_{\text {eff }}\left(v_{\chi}\right)$.

$$
\begin{aligned}
V_{e f f}\left(v_{\chi}\right)= & V_{0}\left(v_{\chi}\right)+\frac{3}{64 \pi^{2}}\left(m_{Z_{2}}^{4}\left(v_{\chi}\right) \ln \frac{m_{Z_{2}}^{2}\left(v_{\chi}\right)}{Q^{2}}-12 m_{Q}^{4}\left(v_{\chi}\right) \ln \frac{m_{Q}^{2}\left(v_{\chi}\right)}{Q^{2}}\right) \\
& +\frac{1}{64 \pi^{2}}\left(m_{h_{2}}^{4}\left(v_{\chi}\right) \ln \frac{m_{h_{2}}^{2}\left(v_{\chi}\right)}{Q^{2}}+2 m_{h^{++}}^{4}\left(v_{\chi}\right) \ln \frac{m_{h^{++}}^{2}\left(v_{\chi}\right)}{Q^{2}}\right) \\
& +\frac{3}{64 \pi^{2}}\left(2 m_{U}^{4}\left(v_{\chi}\right) \ln \frac{m_{U}^{2}\left(v_{\chi}\right)}{Q^{2}}+2 m_{V}^{4}\left(v_{\chi}\right) \ln \frac{m_{V}^{2}\left(v_{\chi}\right)}{Q^{2}}\right) \\
& +\frac{T^{4}}{4 \pi^{2}}\left[F_{-}\left(\frac{m_{h_{2}}\left(v_{\chi}\right)}{T}\right)+2 F_{-}\left(\frac{m_{h^{++}}\left(v_{\chi}\right)}{T}\right)+12 F_{+}\left(\frac{m_{Q}\left(v_{\chi}\right)}{T}\right)\right] \\
& +\frac{3 T^{4}}{4 \pi^{2}}\left[F_{-}\left(\frac{m_{Z_{2}}\left(v_{\chi}\right)}{T}\right)+2 F_{-}\left(\frac{m_{U}\left(v_{\chi}\right)}{T}\right)+2 F_{-}\left(\frac{m_{V}\left(v_{\chi}\right)}{T}\right)\right] .
\end{aligned}
$$

The symmetry breaking scale is $v_{\chi_{0}}$ chosen to be $4 \mathrm{TeV}$ [14, 18] and the masses of three exotic quarks are $m_{Q}$. Therefore, the effective potential can be rewritten as follows

$$
V_{S U(3) \rightarrow S U(2)}^{e f f}=D^{\prime}\left(T^{2}-T_{0}^{\prime 2}\right) v_{\chi}^{2}-E^{\prime} T v_{\chi}^{3}+\frac{\lambda_{T}^{\prime}}{4} v_{\chi}^{4}
$$


The minimum conditions are

$$
V_{e f f}\left(v_{\chi_{0}}\right)=0 ; \quad \frac{\partial V_{e f f}\left(v_{\chi}\right)}{\partial v_{\chi}}\left(v_{\chi_{0}}\right)=0 ; \quad \frac{\partial^{2} V_{e f f}\left(v_{\chi}\right)}{\partial v_{\chi}^{2}}\left(v_{\chi_{0}}\right)=\left.m_{h_{2}}^{2}\left(v_{\chi}\right)\right|_{v_{\chi}=v_{\chi_{0}}},
$$

where

$$
\begin{aligned}
& D^{\prime}= \frac{1}{24 v_{\chi_{0}}^{2}}\left\{6 m_{U}^{2}\left(v_{\chi}\right)+3 m_{Z_{2}}^{2}\left(v_{\chi}\right)+6 m_{V}^{2}\left(v_{\chi}\right)+18 m_{Q}^{2}\left(v_{\chi}\right)+2 m_{h_{2}}^{2}\left(v_{\chi}\right)+2 m_{h^{ \pm}}^{2}\left(v_{\chi}\right)\right\}, \\
& T_{0}^{\prime 2}= \frac{1}{D}\left\{\frac{1}{4} m_{h_{2}}^{2}\left(v_{\chi}\right)-\frac{1}{32 \pi^{2} v_{\chi_{0}}^{2}}\left(6 m_{U}^{4}\left(v_{\chi}\right)+3 m_{Z_{2}}^{4}\left(v_{\chi}\right)+6 m_{V}^{4}\left(v_{\chi}\right)-36 m_{Q}^{4}\left(v_{\chi}\right)\right.\right. \\
&\left.\left.\quad+m_{h_{2}}^{4}\left(v_{\chi}\right)+2 m_{h^{ \pm}}^{4}\left(v_{\chi}\right)\right)\right\}, \\
& E^{\prime}= \frac{1}{12 \pi v_{\chi_{0}}^{3}}\left(6 m_{U}^{3}\left(v_{\chi}\right)+3 m_{Z_{2}}^{3}\left(v_{\chi}\right)+6 m_{V}^{3}\left(v_{\chi}\right)+m_{h_{2}}^{3}\left(v_{\chi}\right)+2 m_{h^{ \pm}}^{3}\left(v_{\chi}\right)\right), \\
& \lambda_{T}^{\prime}= \frac{m_{h_{2}}^{2}\left(v_{\chi}\right)}{2 v_{\chi_{0}}^{2}}\left\{1-\frac{1}{8 \pi^{2} v_{\chi_{0}}^{2} m_{h_{2}}^{2}\left(v_{\chi}\right)}\left[6 m_{V}^{4}\left(v_{\chi}\right) \ln \frac{m_{V}^{2}\left(v_{\chi}\right)}{b T^{2}}+3 m_{Z_{2}}^{4}\left(v_{\chi}\right) \ln \frac{m_{Z_{2}}^{2}\left(v_{\chi}\right)}{b T^{2}}\right.\right. \\
& \quad+6 m_{U}^{4}\left(v_{\chi}\right) \ln \frac{m_{U}^{2}\left(v_{\chi}\right)}{b T^{2}}-36 m_{Q}^{4}\left(v_{\chi}\right) \ln \frac{m_{Q}^{2}\left(v_{\chi}\right)}{b_{F} T^{2}}+m_{h_{2}}^{4}\left(v_{\chi}\right) \ln \frac{m_{h_{2}}^{2}\left(v_{\chi}\right)}{b T^{2}} \\
&\left.\left.\quad+2 m_{h^{ \pm}}^{4}\left(v_{\chi}\right) \ln \frac{m_{h^{ \pm}}^{2}\left(v_{\chi}\right)}{b T^{2}}\right]\right\} .
\end{aligned}
$$

The values of $V_{e f f}\left(v_{\chi}\right)$ at the two minima become equal at the critical temperature

$$
T_{c}^{\prime}=\frac{T_{0}^{\prime}}{\sqrt{1-E^{\prime 2} / D^{\prime} \lambda_{T_{c}^{\prime}}^{\prime}}} .
$$

The problem here is that there are three variables: the masses of $h_{2}, h^{--}$and $Q$. However, for simplicity, following the ansazt in [9], we assume $m_{h_{2}}=X, m_{h^{--}}=m_{Q}=K$ and $m_{Z 2}\left(v_{\chi}\right)=4.821 \mathrm{TeV}$. Note that the contributions from $h_{2}, h^{--}, Z_{2}$, in this phase transition, are $X$ or $K$, different to their contributions in the phase transition $S U(2) \rightarrow U(1)$. In order to have the first phase transition, the phase transition strength must be larger than the unit, i.e., $\frac{v_{\chi c}}{T_{c}^{\prime}} \geq 1$.

In figure 1, we have plotted $K$ as a function of $m_{h_{2}}\left(v_{\chi}\right)$, with $m_{h_{2}}\left(v_{\chi}\right)>1 \mathrm{TeV}$.

According to Fig. 1, if $X$ is larger than $1 \mathrm{TeV}$, the heavy particle masses are in range of few $\mathrm{TeVs}$ in order to have the first order phase transition. In addition, this phase transition can be strong first order.

\section{B. Phase transition $S U(2) \rightarrow U(1)$}

This phase transition dose not involve the exotic quarks and boson $V^{ \pm}$and the contribution from $U^{\mp \mp}$ is equal to $W^{\mp}$. The effective potential of the EWPT $S U(2) \rightarrow U(1)$ is 


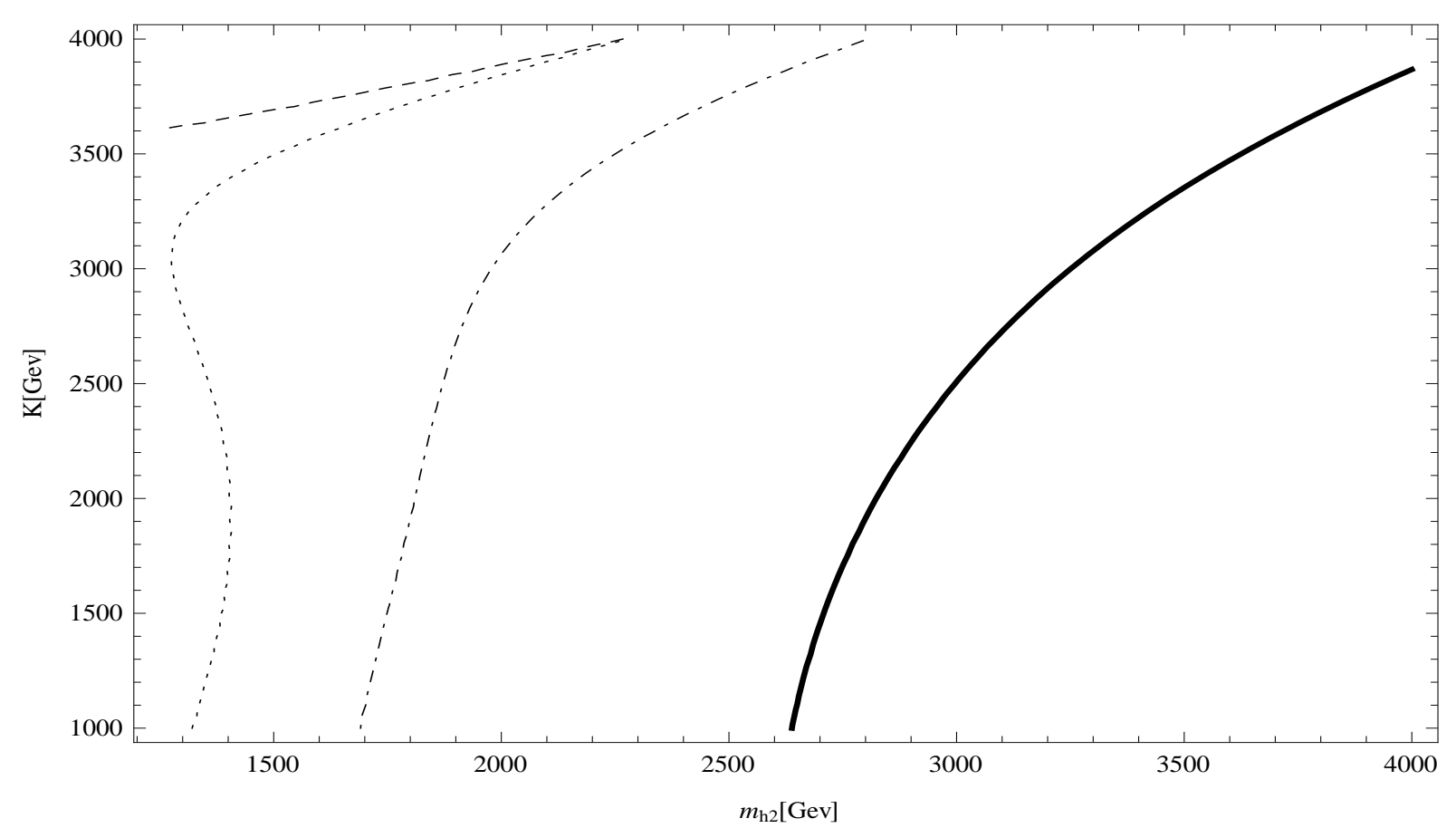

FIG. 1: When $X>1 \mathrm{TeV}$, the solid contours of $2 E^{\prime} / \lambda_{T_{c}^{\prime}}^{\prime}=1$, the dashed-dotted contour: $2 E^{\prime} / \lambda_{T_{c}^{\prime}}^{\prime}=$ 2, the dotted contour: $2 E^{\prime} / \lambda_{T_{c}^{\prime}}^{\prime}=3$, the dashed contour: $2 E^{\prime} / \lambda_{T_{c}^{\prime}}^{\prime}=5$.

$V_{e f f}\left(v_{\rho}\right):$

$$
\begin{aligned}
V_{\text {eff }}\left(v_{\rho}\right)= & V_{0}\left(v_{\rho}\right)+\frac{3}{64 \pi^{2}}\left(m_{Z_{1}}^{4}\left(v_{\rho}\right) \ln \frac{m_{Z_{1}}^{2}\left(v_{\rho}\right)}{Q^{2}}+m_{Z_{2}}^{4}\left(v_{\rho}\right) \ln \frac{m_{Z_{2}}^{2}\left(v_{\rho}\right)}{Q^{2}}\right. \\
& \left.+2 m_{W}^{4}\left(v_{\rho}\right) \ln \frac{m_{W}^{2}\left(v_{\rho}\right)}{Q^{2}}+2 m_{U}^{4}\left(v_{\rho}\right) \ln \frac{m_{U}^{2}\left(v_{\rho}\right)}{Q^{2}}-4 m_{t}^{4}\left(v_{\rho}\right) \ln \frac{m_{t}^{2}\left(v_{\rho}\right)}{Q^{2}}\right) \\
& +\frac{1}{64 \pi^{2}}\left(m_{h_{1}}^{4}\left(v_{\rho}\right) \ln \frac{m_{h_{1}}^{2}\left(v_{\rho}\right)}{Q^{2}}+m_{h_{2}}^{4}\left(v_{\rho}\right) \ln \frac{m_{h_{2}}^{2}\left(v_{\rho}\right)}{Q^{2}}+2 m_{h^{++}}^{4} \ln \frac{m_{h^{++}}^{2}\left(v_{\rho}\right)}{Q^{2}}\right) \\
+ & \frac{T^{4}}{4 \pi^{2}}\left[F_{-}\left(\frac{m_{h_{1}}\left(v_{\rho}\right)}{T}\right)+F_{-}\left(\frac{m_{h_{2}}\left(v_{\rho}\right)}{T}\right)+2 F_{-}\left(\frac{m_{h^{++}}\left(v_{\rho}\right)}{T}\right)\right] \\
+ & \frac{3 T^{4}}{4 \pi^{2}}\left[4 F_{+}\left(\frac{m_{t}\left(v_{\rho}\right)}{T}\right)+F_{-}\left(\frac{m_{Z_{1}}\left(v_{\rho}\right)}{T}\right)+F_{-}\left(\frac{m_{Z_{2}}\left(v_{\rho}\right)}{T}\right)\right. \\
& \left.\quad+2 F_{-}\left(\frac{m_{W}\left(v_{\rho}\right)}{T}\right)+2 F_{-}\left(\frac{m_{U}\left(v_{\rho}\right)}{T}\right)\right] .
\end{aligned}
$$

The minimum conditions are

$$
V_{e f f}\left(v_{\rho_{0}}\right)=0 ; \quad \frac{\partial V_{e f f}}{\partial v_{\rho}}\left(v_{\rho_{0}}\right)=0 ; \quad \frac{\partial V_{e f f}}{\partial v_{\rho}}\left(v_{\rho_{0}}\right)=m_{h_{1}}^{2}+\left.m_{h_{2}}^{2}\left(v_{\rho}\right)\right|_{v_{\rho}=v_{\rho_{0}}} .
$$

From the above minimum conditions, we see that in this EWPT $m_{h_{2}}^{2}\left(v_{\rho}\right)$ plays the role to generate masses of the last heavy particles and $m_{h_{1}}^{2}$ generates masses of the SM particles. 
With the symmetry breaking scale equal to $Q \equiv v_{\rho_{0}}=v_{0}=246 \mathrm{GeV}$, the hightemperature expansion of this potential has the form

$$
V_{e f f}^{R M 331}=D\left(T^{2}-T_{0}^{2}\right) \cdot v_{\rho}^{2}-E T\left|v_{\rho}\right|^{3}+\frac{\lambda_{T}}{4} v_{\rho}^{4},
$$

where

$$
\begin{aligned}
& D=\frac{1}{24 v_{0}^{2}}\left[6 m_{W}^{2}\left(v_{\rho}\right)+6 m_{U}^{2}\left(v_{\rho}\right)+3 m_{Z_{1}}^{2}\left(v_{\rho}\right)+3 m_{Z_{2}}^{2}\left(v_{\rho}\right)\right. \\
& \left.+6 m_{t}^{2}\left(v_{\rho}\right)+m_{h_{1}}^{2}\left(v_{\rho}\right)+m_{h_{2}}^{2}\left(v_{\rho}\right)+2 m_{h^{ \pm}}^{2}\left(v_{\rho}\right)\right], \\
& T_{0}^{2}=\frac{1}{D}\left\{\frac{1}{4}\left(m_{h_{1}}^{2}\left(v_{\rho}\right)+m_{h_{2}}^{2}\left(v_{\rho}\right)\right)-\frac{1}{32 \pi^{2} v_{0}^{2}}\left(6 m_{W}^{4}\left(v_{\rho}\right)+6 m_{U}^{4}\left(v_{\rho}\right)+3 m_{Z_{1}}^{4}\left(v_{\rho}\right)\right.\right. \\
& \left.\left.+3 m_{Z_{2}}^{4}\left(v_{\rho}\right)-12 m_{t}^{4}\left(v_{\rho}\right)+m_{h_{1}}^{4}\left(v_{\rho}\right)+m_{h_{2}}^{4}\left(v_{\rho}\right)+2 m_{h^{ \pm}}^{4}\left(v_{\rho}\right)\right)\right\}, \\
& E=\frac{1}{12 \pi v_{0}^{3}}\left(6 m_{W}^{3}\left(v_{\rho}\right)+6 m_{U}^{3}\left(v_{\rho}\right)+3 m_{Z_{1}}^{3}\left(v_{\rho}\right)+3 m_{Z_{2}}^{3}\left(v_{\rho}\right)\right. \\
& \left.+m_{h_{1}}^{3}\left(v_{\rho}\right)+m_{h_{2}}^{3}\left(v_{\rho}\right)+2 m_{h^{ \pm}}^{3}\left(v_{\rho}\right)\right), \\
& \lambda_{T}=\frac{m_{h_{1}}^{2}\left(v_{\rho}\right)+m_{h_{2}}^{2}\left(v_{\rho}\right)}{2 v_{0}^{2}}\left\{1-\frac{1}{8 \pi^{2} v_{0}^{2}\left(m_{h_{1}}^{2}\left(v_{\rho}\right)+m_{h_{2}}^{2}\left(v_{\rho}\right)\right)}\left[6 m_{W}^{4}\left(v_{\rho}\right) \ln \frac{m_{W}^{2}\left(v_{\rho}\right)}{b T^{2}}\right.\right. \\
& +3 m_{Z_{1}}^{4}\left(v_{\rho}\right) \ln \frac{m_{Z_{1}}^{2}\left(v_{\rho}\right)}{b T^{2}}+3 m_{Z_{2}}^{4}\left(v_{\rho}\right) \ln \frac{m_{Z_{2}}^{2}\left(v_{\rho}\right)}{b T^{2}}+6 m_{U}^{4}\left(v_{\rho}\right) \ln \frac{m_{U}^{2}\left(v_{\rho}\right)}{b T^{2}} \\
& -12 m_{t}^{4}\left(v_{\rho}\right) \ln \frac{m_{t}^{2}\left(v_{\rho}\right)}{b_{F} T^{2}}+m_{h_{1}}^{4}\left(v_{\rho}\right) \ln \frac{m_{h_{1}}^{2}\left(v_{\rho}\right)}{b T^{2}}+m_{h_{2}}^{4}\left(v_{\rho}\right) \ln \frac{m_{h_{2}}^{2}\left(v_{\rho}\right)}{b T^{2}} \\
& \left.\left.+2 m_{h^{ \pm}}^{4}\left(v_{\rho}\right) \ln \frac{m_{h^{ \pm}}^{2}\left(v_{\rho}\right)}{b T^{2}}\right]\right\}
\end{aligned}
$$

The effective potential has two minimum points, the first minimum at $v_{\rho}=0$ and the second one at $v_{\rho_{c}}=\frac{2 E T_{c}}{\lambda_{T_{c}}}$. In the limit $E \rightarrow 0$, we has the second order phase transition. In order to have the first order phase transition, the phase transition strength has to be larger than the unit, i.e., $\frac{v_{\rho_{c}}}{T_{c}} \geq 1$. The critical temperature $T_{c}$ is given by

$$
T_{c}=\frac{T_{0}}{\sqrt{1-E^{2} / D \lambda_{T_{c}}}} .
$$

The equation (16) is self-consistent with the critical temperature because $\lambda_{T_{c}}$ is a function of $T_{c}$. According to the LHC, we take $m_{h_{1}}=125 \mathrm{GeV}$ and put $m_{h_{2}}\left(v_{\rho}\right)=Z, m_{h^{--}}\left(v_{\rho}\right)=Y$ and $m_{Z 2}\left(v_{\rho}\right)=14.53 \mathrm{GeV}$.

In Fig, 2, we show the masses region of $h_{1}$ and $h_{++}$where the necessary condition of the first order phase transition is imposed. According to Fig $[2$, and by the numerical evaluation, the strength of the EWPT is in range, $1 \leq 2 E / \lambda_{T_{c}}<5$. Therefore, in the RM331 we always have the first order phase transition but it is weak (at the $v_{\rho}$ scale). 


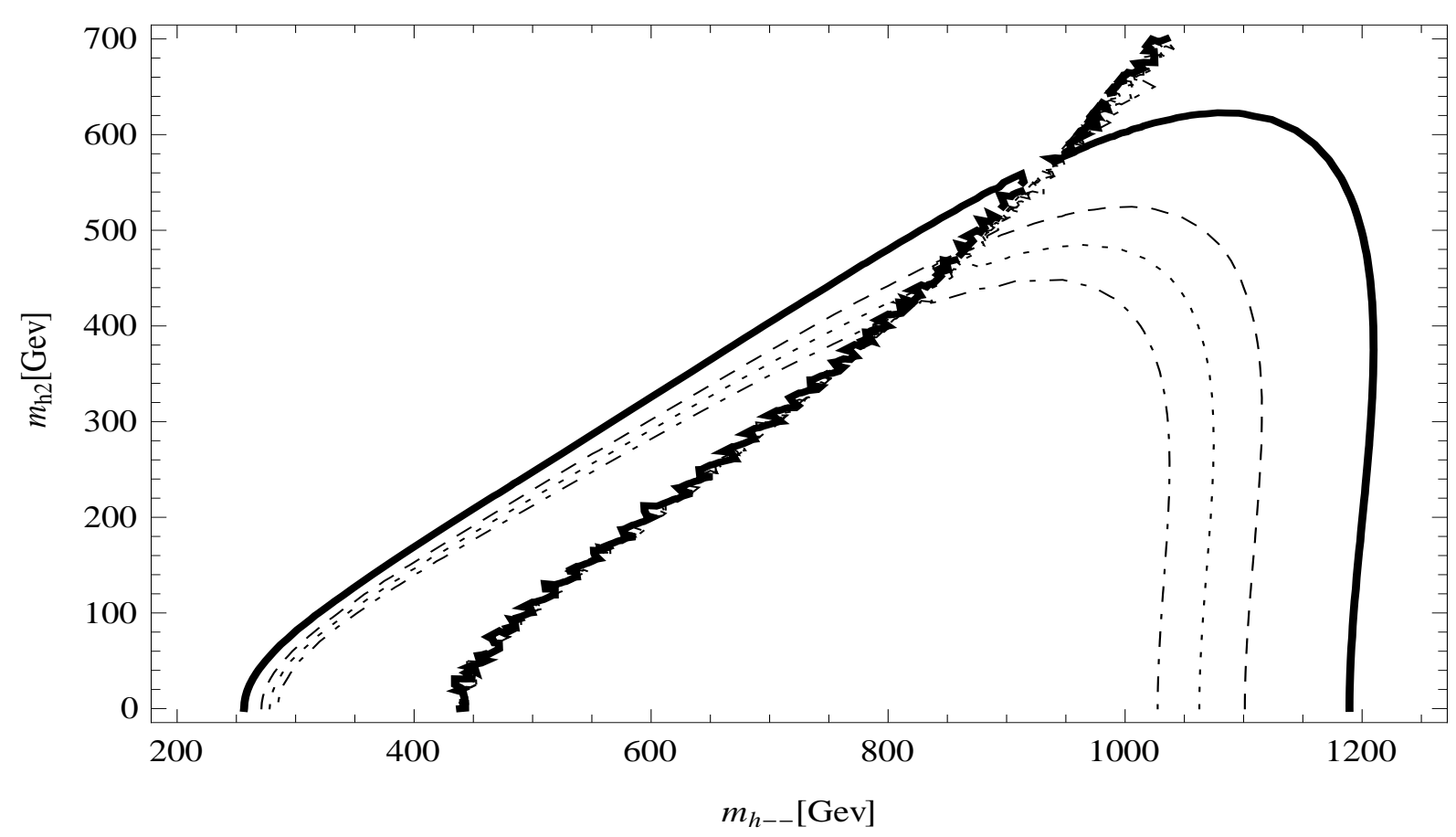

FIG. 2: When the solid contour of $2 E / \lambda_{T_{c}}=1$, the dashed contour: $2 E / \lambda_{T_{c}}=1.1$, the dotted contour: $2 E / \lambda_{T_{c}}=1.15$, the dotted-dashed contour: $2 E / \lambda_{T_{c}^{\prime}}=1.2$

The contributions from new particles (at the first symmetry breaking) make of the first order phase transition that the Standard Model cannot. However, there is one thing special, heavy particles as $U^{ \pm \pm}, h_{2}, h^{--}, Z_{2}$ that contribute only the little part in their total masses.

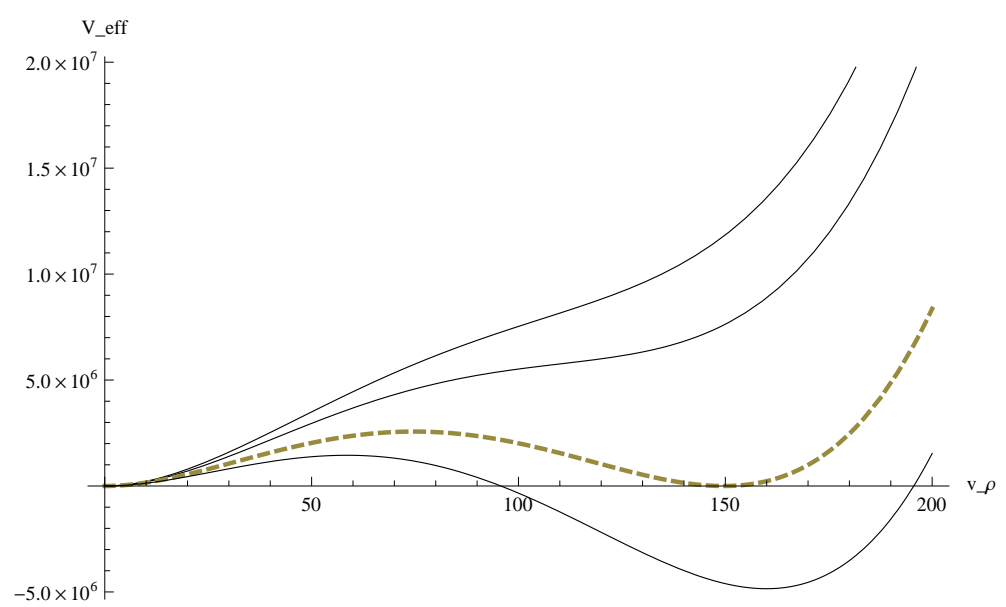

FIG. 3: For example, the effective potential for $2 E / \lambda_{T_{c}}=1$, with $Y=342 \mathrm{GeV}, Z=120.109$ $\mathrm{GeV}$. The critical point at $T_{c}=149.549$ Gev. Solid line: $T=T_{c}$, lines above solid line: $T>T_{c}$, lines under solid line: $T<T_{c}$ 
In Fig. 3, when temperature go close to $T_{c}$, the second minimum slowly formed distinct, ie the phase transition nucleation appears. When temperature go to $T_{c}$, the symmetry breaking phase is turned on and temperature go down $T_{c}$, the system switches to the symmetry breaking phase.

To finish this section, we conclude that the effective potential of this model is different from that of the SM, and it has the contributions from heavy bosons, which plays role of the triggers for the first order phase transition with $m_{h_{1}}=125 \mathrm{GeV}$.

\section{Constraint on mass of charged Higgs boson}

In Section IV, from the phase transition $S U(2) \rightarrow U(1)$, we have derived

$$
200 \mathrm{GeV}<Y=m_{h_{++}}\left(v_{\rho}\right)<1200 \mathrm{GeV}
$$

and

$$
0<Z=m_{h_{2}}\left(v_{\rho}\right)<624 \mathrm{GeV}
$$

Therefore, we get

$$
\begin{aligned}
& (200 \mathrm{GeV})^{2}<\frac{\lambda_{4}}{2} v_{\rho_{0}}^{2}<(1200 \mathrm{GeV})^{2}, \\
& (0 \mathrm{GeV})^{2}<\frac{\lambda_{3}^{2}}{4 \lambda_{2}} v_{\rho_{0}}^{2}<(624 \mathrm{GeV})^{2}
\end{aligned}
$$

Taking into account of the recent Higgs boson mass $(125 \mathrm{GeV})$, combining with the formula (5), we also obtain

$$
\left(\lambda_{1}-\frac{\lambda_{3}^{2}}{4 \lambda_{2}}\right) v_{\rho_{0}}^{2}=(125 \mathrm{GeV})^{2} .
$$

Combination of the equations in (17) and (18) leads to

$$
\begin{aligned}
& 0<\lambda_{1}<6.692 \\
& 0<\frac{\lambda_{3}^{2}}{4 \lambda_{2}}<6.434 \\
& 1.321<\lambda_{4}<47.59
\end{aligned}
$$

From equation (19), leading to $\lambda_{1}, \lambda_{2}, \lambda_{4}$ that must be positive, satisfying the above bound conditions and $3.258 \mathrm{TeV}<m_{h_{++}}<19.549 \mathrm{TeV}$. Thus the mass of the heavy Higgs must be some few $\mathrm{TeVs}$, the electroweak phase transition in this model is the first order phase transition. We hope that the heavy particles will uncover many more new physics. 


\section{CONCLUSION AND OUTLOOKS}

We have used the effective potential at finite temperature to study the structure of the electroweak phase transition in the RM331 model. This phase transition is separated into two phases. The first transition period is $S U(3) \rightarrow S U(2)$, or the symmetry breaking in the energy scale $v_{\chi_{0}}$ (in order to generate masses for the heavy particles and the exotic quarks). The second phase transition is $S U(2) \rightarrow U(1)$ at $v_{\rho_{0}}$, which generates masses for all usual fermions and the SM gauge bosons. The electroweak phase transition in this model (in the scale $v_{\rho}$ ) may be the weakly first order electroweak phase transition with $m_{h_{1}}=125 \mathrm{GeV}$ if the heavy bosons masses are some few TeVs. So this is strong enough to study the baryon asymmetry.

If $Z_{2}$ exists, its mass is some few TeVs, so its contribution to the electroweak phase transition is very large. Therefore, the electroweak phase transition in this model is completely turned on. In other words, the baryon asymmetry problem in this model is directly related to the mass of $Z_{2}$.

The self-interactions of Higgs in this model are more complicated than the SM, because heavy particles involve in both two phase transitions. Thus calculating the quantum corrections can reveal many new physical phenomena and opens up new relations further between Cosmology and Particle Physics. In addition, from the phase transitions, we can get some bounds on the Higgs self-couplings.

Although we only work on the RM331 model, but this calculation can still apply to other 3-3-1 models such as the recent supersymmetric reduced minimal 3-3-1 model [22] and the 3-3-1-1 model [23]. So the 3-3-1 models can have specific advantages more than the SM in explaining the baryon asymmetry problem.

Our next works will calculate the sphaleron rate and CP violations in 3-3-1 models, in order to analysis in details electroweak baryogenesis. In addition, by using the electroweak phase transition or baryogenesis problem, we can predict masses of the heavy particles beyond the SM such as 3-3-1 models. 


\section{Acknowledgment}

The authors thank P. V. Dong and D. T. Huong for critical remarks and stimulating discussions. This research is funded by Vietnam National Foundation for Science and Technology Development (NAFOSTED) under grant number 103.01-2011.63.

[1] V. Mukhanov, Physical Foundations of Cosmology, Cambridge University Press, (2005); A. D. Sakharov, J. Exp. and Theor. Phys. 5: 2427 (1967)

[2] K. Kajantie, M. Laine, K. Rummukainen, and M. Shaposhnikov, Phys. Rev. Lett. 77, 2887 (1996); F. Csikor, Z. Fodor, and J. Heitger, Phys. Rev. Lett. 82, 21 (1999). J. Grant, M. Hindmarsh, Phys. Rev. D. 64. 016002 (2001); M. D’Onofrio, K. Rummukainen, A. Tranberg, JHEP08, 123 (2012).

[3] M. Bastero-Gil, C. Hugonie, S. F. King, D. P. Roy, and S. Vempati, Phys. Lett. B 489, 359 (2000); A. Menon, D. E. Morrissey, and C. E. M. Wagner, Phys. Rev. D 70, 035005 (2004); S. W. Ham, S. K. Oh, C. M. Kim, E. J. Yoo, and D. Son, Phys. Rev. D 70, 075001 (2004).

[4] J. R. Espinosa, T. Konstandin and F. Riva, Nucl. Phys. B 854 (2012) 592.

[5] S. Kanemura, E. Senaha, T. Shindou and T. Yamada, JHEP 1305 (2013) 066.

[6] D. J. H. Chung and A. J. Long, Phys. Rev. D 81, 123531 (2010).

[7] J. M. Cline, G. Laporte, H. Yamashita, S. Kraml, JHEP 0907:040 (2009).

[8] S. Kanemura, Y. Okada, E. Senaha, Phys. Lett. B 606, 361-366 (2005).

[9] S. W. Ham, S-A Shim, and S. K. Oh, Phys. Rev. D 81, 055015 (2010).

[10] G. Barenboim and N. Rius, Phys. Rev. D 58, 065010 (1998).

[11] S. Das, P. J. Fox, A. Kumar, and N. Weiner, JHEP 1011:108 (2010); D. Chung and A. J. Long, Phys. Rev. D. 84, 103513 (2011); M. Carena, N. R. Shaha, and C. E. M. Wagner, Phys. Rev. D 85, 036003 (2012); A. Ahriche and S. Nasri, Phys. Rev. D 85, 093007 (2012); D. Borah and J. M. Cline, Phys. Rev. D 86, 055001 (2013).

[12] New results indicate that particle discovered at CERN is a Higgs boson, http://press.web.cern.ch.

[13] F. Pisano and V. Pleitez, Phys. Rev. D 46, (1992) 410; P. H. Frampton, Phys. Rev. Lett. 69, 
(1992) 2889; R. Foot et al, Phys. Rev. D 47, (1993) 4158.

[14] Alex G. Dias, V. Pleitez, Phys. Rev. D 80056007 (2009).

[15] M. Singer, J. W. F. Valle, and J. Schechter, Phys. Rev. D 22, (1980) 738; R. Foot, H. N. Long, and Tuan A. Tran, Phys. Rev. D 50, (1994) R34, [arXiv: hep-ph/9402243]; J. C. Montero, F. Pisano, and V. Pleitez, Phys. Rev. D 47, (1993) 2918; H. N. Long, Phys. Rev. D 53, (1996) 437; H. N. Long, Phys. Rev. D 54, (1996) 4691; H. N. Long, Mod. Phys. Lett. A13, (1998) 1865 .

[16] C. A. de S. Pires, O. P. Ravinez, Phys. Rev. D 58 (1998) 035008; A. Doff, F. Pisano, Mod. Phys. Lett. A 14 (1999) 1133; Phys. Rev. D 63 (2001) 097903; P.V. Dong, H. N. Long, Int. J. Mod. Phys. A 21 (2006) 6677.

[17] W. A. Ponce, Y. Giraldo and L. A. Sanchez, Phys. Rev. D 67, 075001 (2003); P. V. Dong, H. N. Long, D. T. Nhung and D. V. Soa, Phys. Rev. D 73, 035004 (2006); P. V. Dong and H. N. Long, Adv. High Energy Phys. 2008, 739492 (2008), arXiv:0804.3239]; P. V. Dong, Tr. T. Huong, D. T. Huong, and H. N. Long, Phys. Rev. D 74, 053003 (2006); P. V. Dong, H. N. Long, and D. V. Soa, Phys. Rev. D 73, 075005 (2006); P. V. Dong, H. N. Long, and D. V. Soa, Phys. Rev. D 75, 073006 (2007); P. V. Dong, H. T. Hung, and H. N. Long, Phys. Rev. D 86, $033002(2012)$.

[18] J. G. Ferreira, Jr., P. R. D. Pinheiro, C. A. de S. Pires, and P. S. Rodrigues da Silva, Phys. Rev. D 84, 095019 (2011); V. T. N. Huyen, T. T. Lam, H. N. Long, and V. Q. Phong, arXiv:1210.5833 [hep-ph].

[19] D. Ng, Phys. Rev. D 49, 4805 (1994).

[20] Particle Data Group: J. Beringer et al., Phys. Rev. D 86, 010001 (2012).

[21] See, for examples, D. A. Gutierrez, W. A. Ponce, and L. A. Sanchez, Eur. Phys. J. C 46, 497 (2006); Y. A. Coutinho, V. S. Guimaraes, and A. A. Nepomuceno, arXiv:1304.7907.

[22] T. V. Duong and E. Ma, Phys. Lett. B 316 (1993) 307; J. C. Montero, V. Pleitez, M. C. Rodriguez, Phys. Rev. D6 5 (2002) 035006; R. A. Diaz, R. Martinez, J. A. Rodriguez, Phys. Lett. B 552 (2003) 287; J. C. Montero, V. Pleitez, M. C. Rodriguez, Phys. Rev. D 70 (2004) 075004; P. V. Dong, D. T. Huong, M. C. Rodriguez, H. N. Long, Nucl. Phys. B 772 (2007) 150; P. V. Dong, D. T. Huong, N. T. Thuy, H. N. Long, Nucl. Phys. B 795 (2008) 361; D. T. Huong, L. T. Hue, M. C. Rodriguez, H. N. Long, Nucl. Phys. B 870 (2013) 293; J. G. Ferreira, C. A. de S. Pires, P. S. Rodrigues da Silva, A. Sampieri, e-Print: arXiv:1308.0575; D. T. Binh, 
L. T. Hue, D. T. Huong, H. N. Long, e-Print: arXiv:1308.3085.

[23] P. V. Dong, H. T. Hung, T. D. Tham, Phys. Rev. D 87, 115003 (2013).

[24] At $0^{K}, v_{\rho} \equiv v_{\rho_{0}}=246 \mathrm{GeV}$ and $v_{\chi} \equiv v_{\chi_{0}}=4 \div 5 \mathrm{TeV}$. In this work, we choose $v_{\chi_{0}}=4 \mathrm{TeV}$ 